\title{
Valutazione dei costi di trattamento dei disturbi psicotici con olanzapina, risperidone e neurolettici tipici di un DSM italiano
}

\begin{abstract}
Objective of the present retrospective analysis was to evaluate resources consumption for psychotic patients treatment with olanzapine (OLZ), risperidone (RIS) or typical neuroleptics (NL) during year 2000 in the Dipartimento di Salute Mentale (DSM) of Ravenna. The screening of total number of psychotic patients followed in the Ravenna DSM during year 2000 generated 26 cases treated with OLZ, 22 treated with RIS and 17 treated with NL that were respecting criteria of equivalence for age and illness severity. For these patients we analyzed pharmacological, non pharmacological (medical visits, nurse visits, social assistance, rehabilitative sessions) and hospital interventions during the year of observation, choosing the point of view of the DSM for costs attribution.

The analysis of pharmacological interventions evidentiated a major usage of associated neuroleptics in the RIS and NL groups in respect to OLZ ( $\mathrm{p}<0,05$ OLZ vs RIS and OLZ vs NL), other significant differences were about associated anticholinergics $(\mathrm{OLZ}<\mathrm{NL}, \mathrm{p}<0,05)$ and mood stabilizers $(\mathrm{OLZ}<\mathrm{RIS}, \mathrm{p}<0,05)$. No significant differences for the comparison RIS vs NL.

Non pharmacological interventions analysis showed less numerous nurse interventions in the OLZ group in respect to RIS and NL patients (both comparisons $\mathrm{p}<0,05$ ), minor social assistance activities for OLZ vs RIS $(\mathrm{p}<0,05)$ and more rehabilitative activities in the OLZ and NL groups $(O L Z>N L>R I S, p<0,05$ for all comparisons).

Hospital days during the year of observation in the three groups were 4,42 for OLZ patients, 7,71 for NL patients and 10,95 for RIS patients ( $\mathrm{p}<0,05$ for all comparisons: OLZ vs RIS, OLZ vs NL and RIS vs NL). The sum of pharmacological, non pharmacological and hospital costs (from 8.856.000 to 10.818.000 LIT) didn't generated statistically significant differences even if the OLZ group followed more intense rehabilitative activities $(+71,65 \%$ vs RIS and $+24,41 \%$ vs NL).
\end{abstract}

Farmeconomia e percorsi terapeutici 2001; 2 (4): 217-222

\section{INTRODUZIONE}

I nuovi farmaci antipsicotici hanno un costo per confezione e per DOT (day of therapy, giorno di terapia) significativamente più alto dei neurolettici tipici. Con il presente contributo desideriamo spostare l'attenzione dal semplice costo di acquisizione dei farmaci per allargare le nostre valutazioni ai costi globali del trattamento dei pazienti schizofrenici. In particolare abbiamo voluto verificare se l'impiego di queste più recenti specialità sia capace di cambiare la distribuzione degli oneri tra le varie voci di spesa, specialmente di quelle attinenti all'assitenza territoriale e ospedaliera rispetto alle cure tradizionali, portando ad un vantaggio economico oppure a un migliore impiego delle risorse disponibili.

\section{MATERIALI E METODI}

Il punto di vista adottato per questa analisi è quella della ASL ed in particolare del Dipartimento di Salute Mentale (DSM). Sono stati arruolati, retrospettivamente, 65 pazienti schizofrenici seguiti durante il 2000 presso il DSM di Ravenna e trattati con olanzapina (OLZ) (26 pazienti), risperidone (RIS) (22 pazienti) e neurolettici tipici (NL) (17 pazienti). I pazienti inclusi nell' analisi sono stati selezionati tra tutti i pazienti affetti da schizofrenia e seguiti da strutture del DSM di Ravenna fin dall' inizio del 2000 (gennaio) e per tutto l'anno, in modo che fossero confrontabili per età e gravità della patologia. A questo scopo, poiché non erano disponibili valutazioni della gravità della patologia basate sull'impiego di scale di valutazione a Responsabile del Dipartimento di Salute Mentale presso la ASL di Ravenna

$¥$ Health Outcomes Manager, Eli Lilly Italia S.p.A.

Corrispondenza a: Luca Guidi, Eli Lilly Italia S.p.A., via Gramsci 731, 50019Sesto Fiorentino(FI); tel. 055.425.70.66; E-mail:

guidi_luca@lilly.com 
Tabella 1

Numero di pazienti per gruppo di trattamento, età media, deviazione standard e range

\begin{tabular}{|lcccc|}
\hline & \multicolumn{4}{c|}{ Caratteristiche del campione } \\
& N & ETA $^{\prime}$ & DS & MIN-MAX \\
\hline \hline NL & 17 & 40,82 & 12,47 & $19-63$ \\
\hline \hline OLZ & 26 & 40,12 & 8,61 & $28-63$ \\
\hline \hline RIS & 22 & 41,90 & 9,99 & $28-63$ \\
\hline \hline Differenze non significative & & & \\
\hline
\end{tabular}

clinica (es. Positive and Negative Symptoms Rating Scale), si è utilizzato il criterio di durata della patologia e della conoscenza diretta dei singoli casi da parte dei medici selezionatori. Sempre per rendere il più possibile confrontabili i gruppi OLZ e RIS con il gruppo NL, per quanto attiene alle modalità di trattamento nei gruppi, sono stati esclusi tutti quei pazienti con età maggiore ai 30 anni che, durante il 2000, avessero assunto NL depot per più di 4 mesi. Il trattamento depot, quando stabilizzato, elimina la variabile "compliance" per la terapia così importante per la valutazione dell' outcome di questi pazienti. La terapia quotidiana con neurolettici, infatti, espone al rischio di arbitrarie sospensioni dell' assunzione del farmaco da parte dei pazienti. Ciò può determinare una significativa caduta dell'efficacia del trattamento e un peggioramento delle condizioni cliniche. Poiché gli antipsicotici atipici (OLZ e RIS) non forniscono preparazioni depot si è ritenuto importante confrontare i due gruppi OLZ e RIS con un gruppo di pazienti trattato con NL ad assunzione quotidiana.
A causa dell' alto numero di specialità associate, l'assegnazione dei pazienti ai tre gruppi (OLZ, RIS e NL) è avvenuta considerando come principale il farmaco antipsicotico o neurolettico assunto a dosi piene per i primi 7 mesi dell'osservazione.

I dati relativi agli interventi farmacologici e non farmacologici (ricoveri e assistenza sul territorio) operati sui pazienti nel corso del 2000 sono stati estratti dalle relative cartelle cliniche operando, per l'assistenza territoriale, un doppio check sui diari delle attività infermieristiche $\mathrm{e}$ delle strutture residenziali protette.

In coerenza con il punto di vista adottato per la nostra analisi, abbiamo effettuato l'attribuzione dei costi riferendosi a quelli reali di erogazione delle singole prestazioni e non quelli indicati dai Tariffari Nomenclatori regionali. In particolare l'attribuzione dei costi ospedalieri è stata effettuata basandosi sui dati di costo per giornata di ricovero in SPDC pubblicati da Garattini L. nel 1996 (1) ed attualizzati al 2000, l'attribuzione dei costi per gli interventi non ospedalieri è stata fatta secondo i dati di costo delle prestazioni in

\begin{tabular}{|c|c|c|c|c|c|c|c|}
\hline & $\begin{array}{l}\text { Dosi neurolett. } \\
\text { principale } \\
\text { mg/die }\end{array}$ & $\begin{array}{c}\text { neurolettici } \\
\text { principale }\end{array}$ & $\begin{array}{c}\text { Totale } \\
\text { neurolettici }\end{array}$ & $\begin{array}{c}\text { Totale } \\
\text { antidepressivi }\end{array}$ & $\begin{array}{c}\text { Totale } \\
\text { anticolinergici }\end{array}$ & $\begin{array}{c}\text { Totale } \\
\text { stabilizz. } \\
\text { umore }\end{array}$ & $\begin{array}{l}\text { Totale } \\
\text { farmaci }\end{array}$ \\
\hline & \multirow{2}{*}{ NL } & 262.894 & 1.017.072 & 101.630 & 13.537 & 105.790 & 1.238.030 \\
\hline DS & & 337.155 & 1.385 .352 & 287.154 & 33.572 & 243.977 & 1.404 .935 \\
\hline MEDIA & 13 & 3.665 .860 & 4.120.126 & 203.998 & 6.912 & 114.731 & 4.445.767 \\
\hline DS & UL & 2.819 .283 & 2.761 .670 & 360.502 & 17.944 & 165.995 & 2.889 .082 \\
\hline MEDIA & RIS & 2.075.864 & 2.729.751 & 199.622 & 8.412 & 228.325 & 3.166.110 \\
\hline DS & 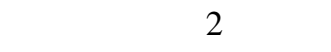 & 1.508 .621 & 1.400 .350 & 452.979 & 26.569 & 372.458 & 1.463 .668 \\
\hline \multicolumn{8}{|c|}{ Significatività: } \\
\hline \multicolumn{2}{|r|}{ OLZ VS RIS } & 0,007 & 0,003 & ns & ns & 0,000 & 0,003 \\
\hline \multicolumn{2}{|r|}{ OLZ VS NL } & 0,000 & 0,006 & ns & 0,005 & ns & 0,004 \\
\hline \multicolumn{2}{|r|}{ RIS VS NL } & 0,000 & $\mathrm{~ns}$ & $\mathrm{~ns}$ & ns & $\mathrm{ns}$ & ns \\
\hline
\end{tabular}

\section{Tabella 2}

Costi per l'acquisizione delle specialità medicinali. La tabella non include i costi della terapia con benzodiazepine perché non rimborsate dal SSN 
psichiatria pubblicati da Fattore G. nel 1997 (2) attraverso il circuito delle farmacie private.

ed attualizzati al 2000, mentre i costi della terapia

farmacologica sono stati individuati attraverso

il Prontuario Farmaceutico Italiano, anno 2000

(3). Nella ASL di Ravenna infatti, nel corso del 2000, la distribuzione diretta non era ancora en-

\section{RISULTATI}

I tre gruppi di pazienti schizofrenici seleziotrata a regime ed i farmaci sono stati distribuiti nati tra quelli che erano in carico al DSM di

\begin{tabular}{|c|c|c|c|c|c|c|c|c|c|}
\hline & \multicolumn{2}{|c|}{ NL } & \multicolumn{2}{|c|}{ OLZ } & \multicolumn{2}{|c|}{ RIS } & \multicolumn{3}{|c|}{ Significatività } \\
\hline & media & ds & media & ds & media & ds & OLZ-RIS & OLZ-NL & RIS-NL \\
\hline $\begin{array}{l}\mathrm{N} \text { visite } \\
\text { specialistiche }\end{array}$ & 9,8 & 6,6 & 8,7 & 8,1 & 10,8 & 8,4 & ns & ns & ns \\
\hline COSTI & 819.688 & 556.449 & 732.896 & 680.540 & 904.306 & 705.630 & ns & ns & ns \\
\hline $\begin{array}{l}\mathrm{N} \text { interventi } \\
\text { infermieristici in }\end{array}$ & 17,8 & 17,7 & 10 & 19,8 & 29,1 & 56,5 & 0,000 & ns & 0,000 \\
\hline COSTI & 52.228 & 52.165 & 29.400 & 58.221 & 85.527 & 166.121 & 0,000 & ns & 0,000 \\
\hline $\begin{array}{l}\mathrm{N} \text { interventi } \\
\text { infermieristici } \\
\text { domiciliari }\end{array}$ & 10,1 & 21,9 & 2,2 & 4,3 & 7,2 & 11,7 & 0,000 & 0,000 & 0,008 \\
\hline COSTI & 404.493 & 879.070 & 87.646 & 171.870 & 288.939 & 468.338 & 0,000 & 0,000 & 0,008 \\
\hline $\begin{array}{l}\mathrm{N} \text { interventi } \\
\text { medici } \\
\text { domiciliari }\end{array}$ & 0,8 & 1,4 & 0,5 & 1 & 0,4 & 1 & ns & ns & $\mathrm{ns}$ \\
\hline COSTI & 153.696 & 288.903 & 92.763 & 206.779 & 82.222 & 202.504 & ns & ns & ns \\
\hline $\begin{array}{l}\mathrm{N} \text { interventi di } \\
\text { psicoterapia }\end{array}$ & 0 & 0 & 1,8 & 7,3 & 1,4 & 5,8 & ns & ns & ns \\
\hline COSTI & 0 & 0 & 271.606 & 1.096 .738 & 218.545 & 872.076 & ns & ns & ns \\
\hline $\begin{array}{l}\mathrm{N} \text { inteventi di } \\
\text { assitenza sociale }\end{array}$ & 2,3 & 5,5 & 2 & 4 & 4,4 & 8,1 & 0,001 & ns & ns \\
\hline COSTI & 109.946 & 262.914 & 95.850 & 195.496 & 213.484 & 386.731 & 0,001 & ns & ns \\
\hline $\begin{array}{l}\mathrm{N} \text { inteventi di } \\
\text { riabilitazione }\end{array}$ & 10,3 & 33,7 & 28,8 & 67,1 & 3,3 & 9,9 & 0,000 & 0,006 & 0,000 \\
\hline COSTI & 263.118 & 862.331 & 737.308 & 1.715 .907 & 83.651 & 255.257 & 0,000 & 0,006 & 0,000 \\
\hline $\begin{array}{l}\mathrm{N} \text { inteventi di } \\
\text { riabilitazione in } \\
\text { struttura } \\
\text { residenziale } \\
\text { protetta }\end{array}$ & 85,9 & 159,6 & 98,2 & 165,1 & 33,2 & 107,4 & 0,049 & ns & ns \\
\hline COSTI & 2.195 .153 & 4.079 .162 & 2.511 .762 & 4.220 .101 & 848.127 & 2.745 .129 & 0,049 & ns & ns \\
\hline $\begin{array}{l}\text { Costo totale } \\
\text { senza } \\
\text { riabilitazione }\end{array}$ & 1.540 .051 & 1.106.611 & 1.310 .161 & 1.304 .746 & 1.793 .023 & 1.302 .036 & ns & ns & $\mathrm{ns}$ \\
\hline $\begin{array}{l}\text { Costo totale } \\
\text { con } \\
\text { riabilitazione }\end{array}$ & 3.998.322 & 5.372 .786 & 4.287 .625 & 4.754 .863 & 2.506 .256 & 3.636 .672 & ns & ns & ns \\
\hline
\end{tabular}

Tabella 3

Costi per l'assistenza territoriale 
Ravenna fin da gennaio 2000 sono omogenei per età e gravità della patologia (Tabella 1$)$. Per quanto riguarda le modalità di trattamento seguite nell'anno di osservazione, per rendere confrontabili i gruppi analizzati, si è cercato di non includere in analisi i pazienti trattati stabilmente con farmaci NL depot. Perciò sono stati esclusi i pazienti con trattamento depot di durata superiore ai 4 mesi nel 2000 e con età superiore ai 30 anni (pazienti in trattamento depot stabilizzato e per questo non confrontabili con pazienti trattati con somministrazione quotidiana degli antipsicotici). Anche così facendo, a causa del criterio età, su 17 pazienti appartenenti al gruppo NL, sei hanno assunto formulazioni depot, cinque per l' intero anno e uno solo per 6 mesi. Esamineremo in sede di discussione il possibile bias indotto da questo fatto nel gruppo NL e le possibili conseguenze sulla valutazione economica.

Dall'analisi dei costi medi per paziente/anno per terapia farmacologica emerge un maggior costo per il ricorso a neurolettici associati nei gruppi RIS (L. 653.887) e NL (L. 754.178) rispetto al gruppo OLZ (L. 454.266) ( $\mathrm{p}<0,05$ sia OLZ vs RIS che OLZ vs NL) (Tabella 2).

Altre differenze significative per i costi di terapia sono emerse relativamente all'associazione di farmaci anticolinergici tra i gruppi OLZ (L . 6.912) e NL (L. 13.537) (p<0,05) e stabilizzatori dell'umore tra i gruppi OLZ (L. 114.731) e RIS (L. 228.325) ( $\mathrm{p}<0,05)$ (Tabella 2).

La dose media di trattamento nel gruppo OLZ è stata di $13 \mathrm{mg} / \mathrm{die}$ (ds: 6), nel gruppo di trattamento RIS $5 \mathrm{mg} / \mathrm{die}$ (ds: 2); non si è calcolata la dose media impiegata nel gruppo di trattamento NL a causa della variabilità di principi farmacologici e preparazioni farmaceutiche impiegate. Nessun'altra differenza statisticamente significativa è emersa per i costi farmacologici

\begin{tabular}{|lcc|}
\hline & $\begin{array}{c}\text { N medio giorni di } \\
\text { ricovero a paziente } \\
\text { nei gruppi }\end{array}$ & $\begin{array}{c}\text { Costo ospedaliero } \\
\text { medio per paziente } \\
\text { (gg) }\end{array}$ \\
\hline \hline $\mathrm{NL}$ & 7,71 & 3.619 .625 \\
\hline OLZ & 4,42 & 2.077 .618 \\
\hline RIS & 10,95 & 5.145 .594 \\
\hline \hline \multicolumn{1}{|c|}{ Significatività: } & & 0,000 \\
OLZ vs RIS & 0,000 & 0,039 \\
\hline OLZ vs NL & 0,039 & 0,019 \\
\hline \hline RIS vs NL & 0,019 & \\
\hline
\end{tabular}

Tabella 4

Costi di ricovero tra i gruppi relativamente all'impiego di terapie associate.

Il computo totale dei costi degli interventi farmacologici, a causa del maggiori prezzi di acquisizione degli atipici, vede il maggior onere economico nel gruppo OLZ (L. 4.445.767), seguito dal gruppo RIS (L. 3.166.110) e quindi dal gruppo NL (L. 1.238.030), con differenze statisticamente significative per i confronti OLZ vs RIS ( $p<0,05)$ e OLZ vs NL ( $<<0,05)$ (Tabella 2). Il computo totale dei costi farmacologici non include i costi di acquisizione delle benzodiazepine perché distribuite in fascia $\mathrm{C}$ e quindi a spese dei pazienti.

Nell'analisi degli interventi non farmacologici il gruppo OLZ ha evidenziato un minor ricorso agli interventi infermieristici in struttura e domiciliari (rispettivamente 10 e 2,2 per paziente/anno con un costo totale di L.117.046) rispetto a quelli richiesti dai gruppi RIS (rispettivamente 29,1 e 7,2 per paziente/ anno con un costo totale di L. 374.466) e NL (rispettivamente 17,8 e 10,1 per paziente/anno con un costo totale di L. 456.721), con $p<0,05$ per tutti i confronti (OLZ vs RIS, OLZ vs NL e RIS vs NL), minor ricorso all'assistenza sociale (OLZ vs RIS, rispettivamente 2 e 4,4 interventi per costi pari a L. 95.850 e L. 213.484 ; p<0,05) e un maggior ricorso ad attività riabilitative $(+71,67 \%$ vs RIS e $+24,41 \%$ vs NL; $p<0,05$ per entrambi) (Tabella 3).

L' analisi dei dati relativi al numero medio di giornate di ricovero per paziente nell'anno di osservazione ha evidenziato differenze statisticamente significative $(\mathrm{p}<0,05)$ tra i gruppi con 4,42 giornate di ricovero nel gruppo OLZ, 7,71 giornate nel gruppo NL e 10,95 giornate nel gruppo RIS (Tabella 4). Nessuna differenza significativa è emersa per i confronti relativi al numero di ricoveri ed al numero di pazienti con ricoveri nei tre gruppi nell' anno di osservazione.

La traduzione in costi della permanenza media per paziente/anno in ospedale nei tre gruppi evidenzia un onere di L. 5.145.594 per RIS, L. 3.619.625 per NL e L. 2.077.618 per OLZ, con $\mathrm{p}<0,05$ per OLZ vs RIS, OLZ vs NL e RIS vs NL) (Tabella 4).

La sommatoria delle singole voci di costo nei tre gruppi, costo globale di trattamento (da L. 8.856.000 a L. 10.818.000), non genera differenze statisticamente significative.

\section{DISCUSSIONE}

Il presente lavoro intende contribuire all'analisi dei costi di trattamento della schizofrenia in Italia prendendo in considerazione sia il periodo di mantenimento, durante il quale i pazienti tipicamente si riferiscono a strutture della Psichiatria territoriale, sia le fasi di ricovero 
ospedaliero che intervengono quando i pazienti dimostrano fasi di riacutizzazione della patologia, per motivi di compliance con la terapia in atto ed efficacia e tollerabilità dei farmaci impiegati. Per il raggiungimento di questo scopo abbiamo svolto una ricerca retrospettiva, di un anno di durata (anno 2000), presso il DSM di Ravenna che comprende sia Servizi Psichiatrici territoriali che reparto SPDC e residenze protette. La scelta di operare attraverso un' analisi retrospettiva è stata determinata dal fatto di voler produrre dati strettamente attinenti alla realtà clinica e procedurale del DSM e dalla disponibilità di una completa documentazione dell'iter terapeutico e degli interventi territoriali ed ospedalieri.

Il primo criterio di selezione dei pazienti entrati a far parte dei tre gruppi di trattamento analizzati è stato quello di raccogliere tutti i casi seguiti presso il DSM di Ravenna per l'intero anno 2000. Il pool di pazienti così selezionati è stato poi vagliato, sulle cartelle cliniche, da personale medico coadiuvato da personale infermieristico, per raccogliere una coorte omogenea per età e gravità della patologia. Si è quindi operata la suddivisione nei tre gruppi OLZ, RIS e NL in base al trattamento di maggior durata nell' arco dell' anno di osservazione. Il periodo medio di trattamento con il farmaco antipsicotico/neurolettico principale nei tre gruppi durante l'anno di osservazione è stato di 275 giorni.

Nel gruppo di trattamento NL, cinque pazienti hanno seguito trattamento depot per l'intero anno e uno solo per 6 mesi. Questo, in cosiderazione del fatto che la terapia con depot consente di eliminare una variabile importante come la compliance (spesso causa di ricoveri o sospensioni arbitrarie del trattamento con conseguente scadimento di efficacia), potrebbe aver influenzato il decorso di questi pazienti nell' anno di osservazione portando ad una pos- sibile sottostima dei costi per l'assistenza territoriale ed ospedaliera nel gruppo NL.

Un altro possibile bias nel confronto OLZ vs NL e RIS vs NL può essere stato indotto dall'esistenza, nel 2000, della nota limitativa 71 bis che consentiva la prescrizione degli atipici ai soli pazienti schizofrenici con dimostrata resistenza/intolleranza al trattamento con neurolettici tipici (3). Questo, nonostante la gravità della patologia fosse paragonabile tra $i$ tre gruppi, potrebbe aver selezionato pazienti tendenzialmente più complessi nei gruppi OLZ e RIS.

Le variabili di assorbimento delle risorse sanitarie che sono state prese in esame hanno compreso tutti gli interventi farmacologici intrapresi nell' anno di osservazione, terapia principale ed associata, interventi delle strutture territoriali (visite specialistiche, visite mediche domiciliari, interventi infermieristici in struttura e domiciliari, colloqui con lo psicologo, assistenza sociale e riabilitazione) ed i ricoveri (questi si sono tutti presentati durante la terapia con il farmaco principale).

I risultati dell'analisi, svoltasi con i criteri sopra indicati, dimostrano che il trattamento con OLZ consente un minor ricorso alle associazioni di neurolettici ed anticolinergici rispetto a NL e di neurolettici e stabilizzatori dell'umore rispetto a RIS ( $p<0,05)$ (Tabella 2$)$. RIS ha consentito invece la sola riduzione dell'associazione di neurolettici nei confronti di NL $(\mathrm{p}<0,05)$ (Tabella2). I costi totali del trattamento farmacologico tuttavia sono mediamente più elevati nel gruppo di trattamento con OLZ a causa del maggior costo di acquisizione del farmaco rispetto a RIS e NL ed a causa del basso costo dei farmaci associati (Tabella 2).

La riduzione degli interventi territoriali che si è generalmente evidenziata nel gruppo di trattamento OLZ rispetto agli altri due gruppi conduce ad un costo totale, per questo tipo di as-

\begin{tabular}{|c|c|c|c|c|}
\hline & NL & OLZ & RIS & $\mathbf{P}$ \\
\hline Costi di ricovero (gg) & 3.619 .625 & 2.077 .618 & 5.145 .594 & $\mathrm{p}<0,05 *$ \\
\hline Costi per interventi sanitari & 3.998 .322 & 4.287 .625 & 2.506 .256 & ns \\
\hline Costi per terapia & 1.238 .030 & 4.445 .767 & 3.166 .110 & $\mathrm{p}<0,05 * *$ \\
\hline Totale & 8.855.977 & 10.811.009 & 10.817 .960 & ns \\
\hline \multicolumn{5}{|c|}{ Sedute di riabilitazione: } \\
\hline Numero medio/anno & 96 & 127 & 36 & $\mathrm{p}<0,05 *$ \\
\hline$\%$ vs OLZ & $-24,41 \%$ & $100,00 \%$ & $-71,65 \%$ & \\
\hline
\end{tabular}

* per OLZ vs RIS e OLZ vs NL e RIS vs NL

** per OLZ vs RIS e OLZ vs NL

Tabella 5

Costi totali di trattamento 
sistenza, che è tendenzialmente inferiore se si escludono dal computo le sessioni di riabilitazione. Vogliamo infatti considerare queste non come un intervento diretto al semplice controllo della sintomatologia, ma come un plus assistenziale che, consentito da un trattamento farmacologico adeguato, permette il recupero dei pazienti e si oppone alla progressione del decadimento cognitivo (4-8). Tuttavia, anche computando i costi relativi al maggior numero di giornate di riabilitazione che hanno potuto seguire i pazienti in trattamento con OLZ rispetto a quelli trattati con RIS o NL $(+24,4 \%$ vs NL e $+71,65 \%$ vs RIS, $p<0,05$ per entrambi), i costi totali per l' assistenza territoriale non giungono a differenze statisticamente significative (Tabella 3).

Nel calcolo economico dell'impatto esercitato dai pazienti affetti da schizofrenia sulle strutture sanitarie, la voce di maggior peso è sicuramente rappresentata dai ricoveri ospedalieri (4,5,7-9). Anche la nostra analisi conferma i dati fino ad ora pubblicati in letteratura (4,5,7-9), evidenziando che i costi ospedalieri rappresentano il $47,6 \%$ della spesa totale media per paziente/anno nel gruppo RIS, il 40,9\% nel gruppo NL ed il 19,2\% nel gruppo OLZ. In particolare, considerando il numero medio di giornate di ricovero per paziente nei tre gruppi, emerge che i pazienti trattati con OLZ effettuano un numero mediamente più basso di giornate di ricovero per anno ( $\mathrm{p}<0,05$ sia vs RIS che vs NL), mentre i pazienti trattati con RIS ne effettuano un numero mediamente più alto ( $p<0,05$ sia vs NL che vs OLZ) (Tabella 4).

La somma totale dei costi farmacologici e non farmacologici (ospedalieri e territoriali), nei tre gruppi di trattamento, porta a totali non significativamente diversi.

In conclusione, la nostra analisi evidenzia che il trattamento con gli antipsicotici atipici non è economicamente più oneroso di quello con neurolettici tipici nel nostro paese.

Abbiamo sottolineato anche che i maggiori costi di acquisizione farmaco principale nel gruppo di trattamento OLZ, coerentemente con quanto già pubblicato di esperienze internazionali e non (4-14), sono compensati dal minor ricorso a ricoveri ed interventi territoriali. In particolare, ad un costo totale di trattamento ed assistenza paragonabile, OLZ ha consentito di effettuare un numero di giornate di riabilitazione significativamente superiore a quello effettuato con NL e RIS (Tabelle 3, 4 e 5).

\section{BIBLIOGRAFIA}

1. Garattini L, Zanelli E, Vesconi D.Analisi dei costi per divisione su un campione di nove presidi ospedalieri. ASI1996; 10: 33-37.

2. Fattore G, Percudani M, Pugnoli C, Contini A. I costi delle prestazioni in psichiatria: un' analisi condotta in un Centro Psico-Sociale (CPS) pubblico in Lombardia. Epidemiologia e Psichiatria Sociale 1997; 6(2): 139-147.

3. L' Informatore Farmaceutico 2000. OEMF, 61a edizione.

4. Berardi D, Dell' Atti M, Guidi L, Russo F, Vagnini V. Costo del trattamento con olanzapina nei Servizi di Salute Mentale. Atti Congresso SIP 2000, pag. 820.

5. Hamilton SH et al. Functional outcomes in schizophrenia: a comparison od olanzapine and haloperidol in an European sample. Int. Clin. Psychopharmacology 2000; 15(5): 245-255.

6. Revicki DA, Genduso LA, Hamilton SH, et al. Olanzapine versus haloperidol in the treatment of schizophrenia and other related psychotic disorders: quality of life and clinical outcomes of a randomized clinical trial. Quality of Life Research 1999; 8:417-426.

7. Tran PV, Delva MA, Tollefson GD, et al. Extrapyramidal symptoms and tolerability of olanzapine versus haloperidol in the acute treatment of schizophrenia. Journal of Clinical Psychiatry 1997;58:205-11.

8. Grainger D. et al. Resource use and quality of life of olanzapine compared with risperidone: results from an international randomized clinical trial. European Neuropsychopharmacology 1998; 8(suppl.A): s225-s226.

9. Edgell ET et al. Olanzapine vs risperidone, a perspective comparison of clinical and economic outcomes in schizophrenia. Pharmacoeconomics 2000; 18(6): 567-579.

10. Sanger TM et al. Olanzapine vs haloperidol treatment in first-episode psychosis. Am. J. Psychiatry, 1999, 156(1): 7987.

11. Sacristan JA, Gomez JC, Carulla S.Analisis coste-efectividad de olanzapina frente a haloperidol en el trattamiento de la esquizofrenia en Espagna. Actas Luso-Esp Neurol. Psiquiatr., 1997, 25(4): 225-234.

12. Sacristan JA, Gomez JC, Martin J et al. Pharmacoeconomic assessment of olanzapine in the treatment of refractory schizophrenia based on a pilot clinical study. Clin. Drug Invest., 1998, 15(1):29-35.

13. Foster RH, Goa KL. Olanzapine. A pharmacoeconomic review of its use in schizophrenia. Pharmacoeconomics, 1999, 15(6): 611-640.

14. Almond S, O'Donnell O. Cost analysis of the treatment of schizophrenia in the UK, a comparison of olanzapine and haloperidol. Pharmacoeconomics, 1998, 13(5 pt 2): 575-588. 